\title{
Software Reliability Growth Models with Log-logistic Testing-Effort Function: A Comparative Study
}

\author{
N. Ahmad and Md. Zafar Imam \\ University Department of Statistics and Computer Applications \\ T. M. Bhagalpur University, Bhagalpur-812007, India
}

\begin{abstract}
Software reliability growth model is one of the basic techniques to assess software reliability quantitatively and it provides the essential information for software development activities. In this paper we compare the predictive capability of popular software reliability growth models (SRGM), such as exponential growth, delayed S-shaped growth and inflection S-shaped growth models. We first review the loglogistic testing-effort function and also discuss exponential type and S-shaped types SRGM with log-logistic testingeffort. We analyze the real data applications and compare the predictive capability of these SRGM. The experimental results reveal that inflection S-shaped type SRGM has better prediction capability as compare to exponential type SRGM
\end{abstract}

\section{Keywords}

Software reliability growth models, testing-effort function, software testing, non-homogeneous Poisson process, estimation methods.

\section{INTRODUCTION}

Software reliability is defined as the probability of failure-free operation of a computer program for a specified time in a specified environment (Musa et al., 1987; Lyu, 1996) and is a key factor in software development process. Numerous software reliability growth models (SRGMs) have been developed during the last three decades and they have applied successfully in practice to improve software reliability (Musa et al., 1987; Xie, 1991; Lyu, 1996; Pham, 2000).

In the past years, several SRGMs based on NHPP which incorporates the testing-effort functions (TEF) have been proposed by many authors (Yamada et al., 1984; 1986; 1993; Yamada and Ohtera, 1990; Huang et al., 2007; Kuoet al., 2001; Ahmad et al. 2008; 2010; Bokhari and Ahmad, 2006; Quadriet al., 2011). Currently, Ahmad et al. (2010a; 2011) also proposed a new SRGM with Log-logistic testing-effort functions to predict the behavior of failure and fault of software.

This paper first reviews the Log-logistic testing-effort function and then incorporates the Log-logistic testing-effort function into exponential and S-shaped NHPP growth models. Actual data applications are analyzed and the predictive capability of these two SRGM is compared.

\section{REVIEW OF LOG-LOGISTIC TESTING-EFFORT}

Recently, Bokhari and Ahmad, 2006 and Ahmad et al. (2010a; 2011) proposed Log-logistic testing-effort function to predict the behavior of failure and fault of a software product. They have shown that Log-logistic testing-effort function is very suitable and more flexible testing resource for assessing the reliability of software products. The cumulative testingeffort expenditure consumed in $(0, t]$ is depicted in the following:

$W(t)=\alpha\left[1-\left\{1+(\beta t)^{\delta}\right\}^{-1}\right]=\alpha\left[(\beta t)^{\delta} /\left(1+(\beta t)^{\delta}\right)\right], t>0$.

Therefore, the current testing-effort expenditure at testing $t$ is given by:

$w(t)=\left[\alpha \beta \delta(\beta t)^{\delta-1}\right] /\left[1+(\beta t)^{\delta}\right]^{2}, t>0, \alpha>0, \beta>0, \delta>0$.

where $\alpha$ is the total amount of testing-effort consumption required by software testing, $\beta$ is the scale parameter, and $\delta$ is the shape parameter.

The testing-effort $w(t)$ reaches its maximum value at time

$$
t_{\max }=\frac{1}{\beta}\left(\frac{\delta-1}{\delta+1}\right)^{\frac{1}{\delta}}
$$

\section{SOFTWARE RELIABILITY GROWTH MODELS WITH TESTING EFFORT}

In this section, we have discussed three basic software reliability growth models, such as Exponential growth model, delayed S-shaped, and inflection S-shaped growth models. These models have been shown to be very useful in fitting software failure data.

\subsection{Exponential Type SRGM with Log- logistic testing-effort}

The exponential growth model proposed by Goel and Okumoto (1979) has been considered for comparative study. Based on the basic assumptions, if the number of detected errors by the current testing-effort expenditures is proportional to the number of remaining errors, then we obtain the following differential equation (Yamada and Osaki, 
1985; Yamada et al., 1986; 1993; Yamada and Ohtera, 1990; Bokhari and Ahmad, 2006):

$$
\frac{d m(t)}{d t} / w(t)=b[a-m(t)], a>0,0<b<1,
$$

where $m(t)$ represent the expected mean number of errors detected in time $(0, t]$ which is assumed to be a bounded non-decreasing function of $t$ with $m(0)=0, w(t)$ is the current testing-effort expenditure at time $t, a$ is the expected number of initial error in the system, and $b$ is the error detection rate per unit testing-effort at time $t$. Solving the above differential equation, we have

$$
m(t)=a\left(1-e^{-b W(t)}\right)
$$

Substituting $W(t)$ from (1), we get

$$
m(t)=a\left(1-e^{-b \alpha\left(\frac{(\beta t)^{\delta}}{1+(\beta t)^{\delta}}\right)}\right) .
$$

This is an NHPP model with mean value function considering the Log-logistic testing-effort expenditure.

\subsection{Delayed S-shaped Type SRGM with Log-logistic testing-effort}

Delayed S-shaped NHPP model was proposed by Yamada et al. (1984). Later, Huang et al. (2007) modified this model and incorporated the logistic testing-effort in an NHPP growth model. On the basis of assumptions (Huang et al., 2007), we obtain the following differential equation:

$$
\frac{d m(t)}{d t} \times \frac{1}{w(t)}=\phi(t)(a-m(t)),
$$

where $\phi(t)=\frac{r^{2} t}{1+r t}, r(>0)$ is the inflection rate and represents the proportion of independent errors present in the software. Solving (6) with the initial condition that, at $t=0, W(t)=0, m(t)=0$, we obtain the mean value function

$$
m(t)=a\left[1-(1+r W(t)) e^{-r W(t)}\right] .
$$

Substituting $W(t)$ from (1), we get

$$
m(t)=a\left[1-\left(1+r \alpha(\beta t)^{\delta} /\left(1+(\beta t)^{\delta}\right)\right) e^{-r \alpha(\beta t)^{\delta} /\left(1+(\beta t)^{\delta}\right)}\right]
$$

\subsection{Inflection S-shaped Type SRGM with Log-logistic testing-effort}

Ohba $(1984 ; 1984 a)$ raised the inflection S-shaped NHPP model. Later, Ahmad et al. (2011) modified the inflection Sshaped model and incorporated the Log-logistic testing-effort in an NHPP growth model.

On the basis of assumptions, if the error detection rate with respect to current testing-effort expenditures is proportional to the number of detectable errors in the software and the proportionality increases linearly with each additional error removal, we obtain the following differential equation:

$$
\frac{d m(t)}{d t} \times \frac{1}{w(t)}=\phi(t)(a-m(t))
$$

where

$$
\phi(t)=b\left[r+(1-r) \frac{m(t)}{a}\right]
$$

$r(>0)$ is the inflection rate and represents the proportion of independent errors present in the software. Solving (9) with the initial condition that, at $t=0, W(t)=0, m(t)=0$, we obtain the mean value function:

$$
m(t)=\frac{a\left[1-e^{-b W(t)}\right]}{1+((1-r) / r) e^{-b W(t)}} .
$$

Substituting $W(t)$ from (1), we get

$$
m(t)=\frac{a\left[1-e^{-b \alpha\left[(\beta t)^{\left.\delta /\left(1+(\beta t)^{\delta}\right)\right]}\right]}\right.}{1+((1-r) / r) e^{-b \alpha\left[(\beta t)^{\delta} /\left(1+(\beta t)^{\delta}\right)\right]}} .
$$

\section{ESTIMATION OF PARAMETERS BY LEAST SQUARE METHOD}

Least Square Estimation (LSE) technique is used to estimate the model parameters (Musa et al., 1987; Musa, 1999; Lyu, 1996). It minimizes the sum of squares of the deviations between what we expect and what we actually observe. That is, we can estimate the parameters $\alpha, \beta$, and $\delta$ of the logistic testing function in (1) and the parameters $a, b$, and $r$ given in (5), (8), and (11) by the method of least squares. These estimates can be obtained by minimizing the following:

$$
\begin{aligned}
& S(\alpha, \beta, \delta)=\sum_{k=1}^{n}\left[W_{k}-W\left(t_{k}\right)\right]^{2} \\
& S(a, b, r)=\sum_{k=1}^{n}\left[m_{k}-m\left(t_{k}\right)\right]^{2}
\end{aligned}
$$

where $W_{k}$, is the cumulative testing effort really consumed in time $\left(0, t_{k}\right]$ and $W\left(t_{k}\right)$ is the cumulative testing effort estimated by the logistic testing function in (1). The $m_{k}$ is the cumulative number of detected errors in a given time 
interval $\left(0, t_{k}\right]$ and $m\left(t_{k}\right)$ is the estimated cumulative

number of detected errors in (5), (8), and (11).

Table I: Summary of studied actual data sets.

\begin{tabular}{|l|l|l|l|l|}
\hline Data Set & References & Errors Removed & Observation Period & Software Project \\
\hline DS1 & Ohba (1984) & 328, after 3.5 years: 188 & 19 weeks & $\begin{array}{l}\text { PL/1 application software, } \\
\text { Execution Time: 47.65CPU hours, } \\
\text { Size: } 1317000 \text { line of code }\end{array}$ \\
\hline DS2 & Musa et al. (1987) & 136, after a long time of testing: 358 & 21 weeks & $\begin{array}{l}\text { Rome Air Development Center } \\
\text { Project, Execution Time: 25.3 CPU } \\
\text { hours, Size: 21700 line of code }\end{array}$ \\
\hline
\end{tabular}

\section{COMPARISON OF PREDICTIVE CAPABILITY}

Least Square estimation (LSE) techniques are used to estimate the model parameters (Musa et al., 1987; Musa, 1999; Lyu, 1996; Ahmad et al., 2008; 2010; 2011). The parameters of the SRGM are estimated based upon the data given in Table I.

In order to compare predictive capability of exponential growth model and inflection S-shaped model with LLTEF, experiments on two actual software failure data are performed. The description of the data sets is given in Table I.

\subsection{Predictive Validity}

The predictive validity is defined (Musa et al., 1987; Musa, 1999 ) as the capability of the model to predict future failure behavior from present and past failure behavior. Assume that we have observed $q$ failures by the end of test time $t_{q}$. We use the failure data up to time $t_{e}\left(\leq t_{q}\right)$ to determine the parameters of $m(t)$. Substituting the estimates of these parameters in the mean value function yields the estimate of the number of failures $\hat{m}\left(t_{q}\right)$ by $t_{q}$. The estimate is compared with the actually observed number $q$. This procedure is repeated for various values of $t_{e}$. The ratio

$$
\frac{\hat{m}\left(t_{q}\right)-q}{q}
$$

is called the relative predictive error (RPE). Values close to zero for RPE indicate more accurate prediction and hence a better model. We can visually check the predictive validity by plotting the relative error for normalized test time $t_{e} / t_{q}$.

DS 1: Table II lists the comparisons of exponential growth model and inflection S-shaped growth model with LLTEF. Results reveal that the inflection S-shaped growth model with LLTEF has better performance. We compute the relative error in prediction of exponential growth model and inflection $\mathrm{S}$ -

shaped growth model with LLTEF for this data set. Results are presented in Tables III and IV. Figures 1 and 2 show the relative error plotted against the percentage of data used (that is, $t_{e} / t_{q}$ ). Figures 1,2 and Tables II, III, IV reveal that the future behavior well as compare to exponential growth model.

Table II: Comparison results of exponential model and inflection S-shaped model

\begin{tabular}{|l|c|c|c|c|c|}
\hline \multicolumn{1}{|c|}{ Model } & $\boldsymbol{a}$ & $\boldsymbol{r}$ & $\boldsymbol{b}$ & $\begin{array}{c}\text { AE } \\
(\boldsymbol{\%})\end{array}$ & MSE \\
\hline $\begin{array}{l}\text { Exponential } \\
\text { model with } \\
\text { LLTEF }\end{array}$ & 565.73 & & 0.0196 & 58.02 & 116.74 \\
\hline $\begin{array}{l}\text { Inflection } \\
\text { S-shaped } \\
\text { model with } \\
\text { LLTEF }\end{array}$ & 385.63 & 0.37 & 0.0622 & 7.54 & 87.69 \\
\hline
\end{tabular}

Table III: Relative error of exponential growth model

\begin{tabular}{|c|c|}
\hline $\begin{array}{c}\text { Percentage of data used } \\
\text { (te/tq\%) }\end{array}$ & Relative Error \\
\hline 32 & 0.206280 \\
37 & 0.052395 \\
42 & -0.006591 \\
47 & 0.079641 \\
53 & 0.028479 \\
58 & -0.014245 \\
63 & -0.032523 \\
68 & -0.047251 \\
74 & -0.065585 \\
79 & -0.035465 \\
84 & -0.011988 \\
89 & 0.001997 \\
95 & 0.025681 \\
100 & 0.053109 \\
\hline
\end{tabular}

Table IV: Relative error of inflection S-shaped model

\begin{tabular}{|c|c|}
\hline $\begin{array}{c}\text { Percentage of data used } \\
\text { (te/tq\%) }\end{array}$ & Relative Error \\
\hline 32 & 0.15391 \\
37 & 0.02753 \\
42 & -0.01371 \\
47 & 0.08569 \\
53 & 0.04369 \\
58 & 0.00592 \\
63 & -0.01043 \\
\hline
\end{tabular}




\begin{tabular}{|c|c|}
\hline 68 & -0.02605 \\
74 & -0.04782 \\
79 & -0.02243 \\
84 & -0.00598 \\
89 & -0.00084 \\
95 & 0.01245 \\
100 & 0.02793 \\
\hline
\end{tabular}

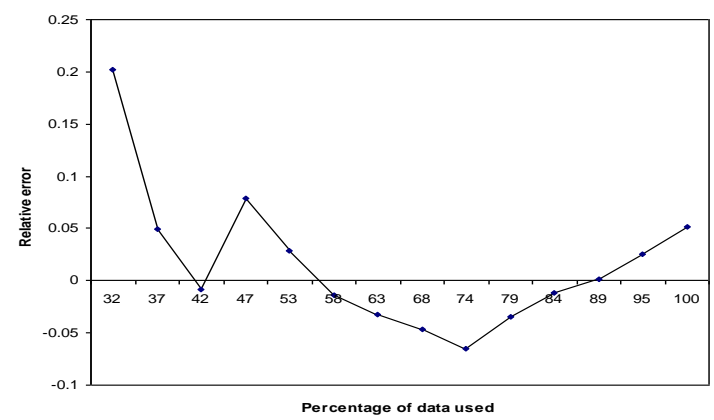

Figure 1: Predictive Relative Error Curve of exponential growth model with LLTEF

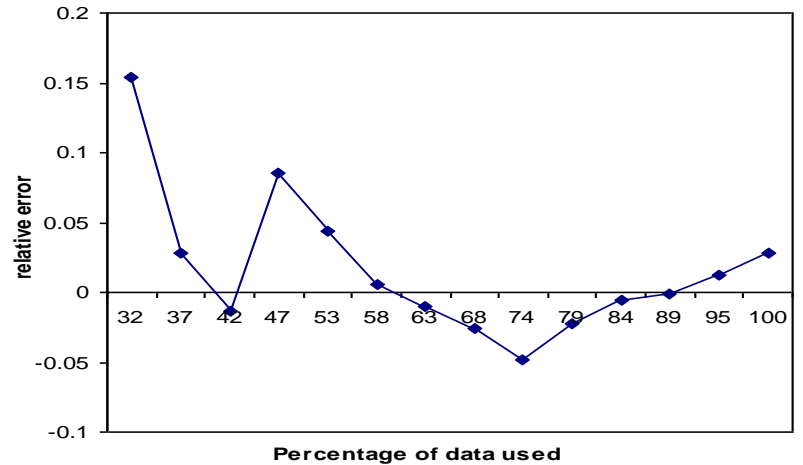

Figure 2: Predictive Relative Error Curve of inflection Sshaped growth model with LLTEF

DS 2: Table V shows the comparisons of exponential model and inflection S-shaped model with LLTEF. Results in the table reveal that the inflection S-shaped model has better performance for this data set. The relative error in prediction is calculated for exponential growth model and inflection Sshaped growth model with LLTEF and the results are presented in Tables VI and VII. These results are shown graphically in Figures 3 and 4. Finally, from the Figures and Tables, it can be concluded that the inflection S-shaped model gets reasonable prediction as compare to exponential model.

Table V. Comparison results of exponential model and inflection S-shaped model

\begin{tabular}{|c|c|c|c|c|c|}
\hline Model & $\boldsymbol{a}$ & $\boldsymbol{r}$ & $\boldsymbol{b}$ & $\begin{array}{c}\text { AE } \\
(\boldsymbol{\%})\end{array}$ & MSE \\
\hline $\begin{array}{l}\text { Exponential } \\
\text { model with } \\
\text { LLTEF }\end{array}$ & 133.28 & & 0.1571 & 29.11 & 100.18 \\
\hline
\end{tabular}

\begin{tabular}{|l|l|l|l|l|l|}
\hline $\begin{array}{l}\text { Inflection } \\
\text { S-shaped } \\
\text { model with }\end{array}$ & 161.02 & 168.37 & 0.0011 & 14.36 & 75.19 \\
LLTEF & & & & & \\
\hline
\end{tabular}

Table VI. Relative error of exponential growth model

\begin{tabular}{|c|c|}
\hline $\begin{array}{c}\text { Percentage of data } \\
\text { used (te/tq\%) }\end{array}$ & Relative Error \\
\hline 24 & -0.979329 \\
29 & -0.949302 \\
33 & -0.869559 \\
38 & -0.852834 \\
43 & -0.814512 \\
48 & -0.642782 \\
52 & -0.507276 \\
57 & -0.201915 \\
62 & -0.019577 \\
67 & 0.083816 \\
71 & 0.081938 \\
76 & 0.102183 \\
81 & 0.072370 \\
86 & -0.008853 \\
90 & -0.029318 \\
95 & -0.037738 \\
100 & -0.037529 \\
\hline
\end{tabular}

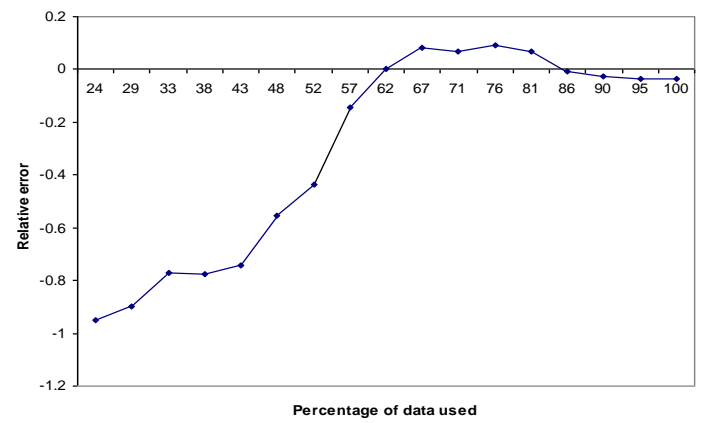

Figure 3: Predictive Relative Error Curve of exponential model

Table VII. Relative error of inflection S-shaped model

\begin{tabular}{|c|c|}
\hline $\begin{array}{c}\text { Percentage of data used } \\
\text { (te/tq\%) }\end{array}$ & Relative Error \\
\hline 24 & -0.97151 \\
29 & -0.93019 \\
33 & -0.82091 \\
38 & -0.79932 \\
43 & -0.75063 \\
48 & -0.53194 \\
52 & -0.38056 \\
57 & -0.05245 \\
\hline
\end{tabular}




\begin{tabular}{|c|c|}
\hline 62 & 0.08967 \\
67 & 0.13232 \\
71 & 0.08096 \\
76 & 0.07707 \\
81 & 0.04448 \\
86 & -0.02737 \\
90 & -0.03669 \\
95 & -0.03449 \\
100 & -0.02535 \\
\hline
\end{tabular}

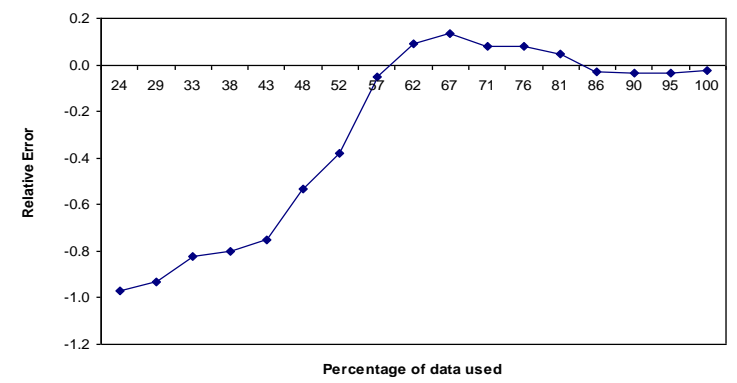

Figure 4: Predictive Relative Error Curve of inflection Sshaped model

\section{CONCLUSION}

This paper discussed exponential type and S-shaped type SRGMs with Log-logistic testing-effort. We estimated the parameters and analyzed the predictive capability of exponential growth and S-shaped growth models with LLTEF for the actual data applications. We then compared its predictive capability. The findings reveal that inflection Sshaped type SRGM has better prediction capability as compare to exponential type SRGM.

\section{ACKNOWLEDGMENTS}

The authors are thankful to the Editor and the Reviewers for their valuable comments and suggestions to improve the manuscript.

\section{REFERENCES}

[1] Ahmad, N., Bokhari, M. U., Quadri, S. M. K. and Khan, M. G. M. (2008), "The Exponetiated Weibull Software Reliability Growth Model with Various Testing-Efforts and Optimal Release Policy: A Performance Analysis", International Journal of Quality and Reliability Management, Vol. 25 (2), 211-235.

[2] Ahmad, N., Khan, M. G. M. and Islam, S. F. (2012), "Optimal Allocation of Testing Resource for Modular Software based on Testing-Effort Dependent Software Reliability Growth", in Proceedings of the third International Conference on Computing Communication \& Networking Technologies (ICCCNT-2012), IEEE Computer Society, Coimbatore, India, pp. 1-7.

[3] Ahmad, N., Khan, M.G.M and Rafi, L.S. (2011), "Analysis of an Inflection S-shaped Software Reliability Model Considering Log-logistic Testing-Effort and Imperfect Debugging", International Journal of Computer Science and Network Security, Vol. 11 (1), pp. $161-171$
[4] Ahmad, N., Khan, M.G.M and Rafi, L.S. (2010), "A Study of Testing-Effort Dependent Inflection S-Shaped Software Reliability Growth Models with Imperfect Debugging", International Journal of Quality and Reliability Management, Vol. 27 (1), pp. $89-110$.

[5] Ahmad, N., Khan, M.G.M and Rafi, L.S. (2010a), "Software Reliability Modeling Incorporating LogLogistic Testing-Effort with Imperfect Debugging", in Proceedings of the International Conference on Modeling, Optimization and Computing (ICMOC-2010), Durgapur, India, Published by American Institute of Physics, pp. $651-657$

[6] Ahmad, N., Quadri, S.M.K. and Razeef, M. (2011), "Comparison of Predictive Capability of Software Reliability Growth Models with Exponentiated Weibull Distribution", International Journal of Computer Applications, DOI 10.5120/1949-2607, Vol. 15 (6), pp. 40-43.

[7] Bokhari, M.U. and Ahmad, N. (2006), “Analysis of a Software Reliability Growth Models: the Case of Loglogistic Test-effort Function", in: Proceedings of the $17^{\text {th }}$ IASTED International Conference on Modeling and Simulation (MS'2006), Montreal, Canada, pp. 540-545.

[8] Goel, A.L. and Okumoto, K. (1979), "Time Dependent Error-detection Rate Model for Software Reliability and Other Performance Measures", IEEE Transactions on Reliability, Vol. R- 28, No. 3, pp. 206-211.

[9] Huang, C.Y., Kuo, S.Y. and Lyu, M.R. (2007), “An Assessment of Testing-effort Dependent Software Reliability Growth Models", IEEE Transactions on Reliability, Vol. 56, no.2, pp. 198-211.

[10] Kuo, S.Y., Hung, C.Y. and Lyu, M.R. (2001), "Framework for Modeling Software Reliability, Using Various Testing-efforts and Fault Detection Rates", IEEE Transactions on Reliability, Vol. 50, no.3, pp 310320 .

[11] Lyu, M.R. (1996), Handbook of Software Reliability Engineering, McGraw- Hill.

[12] Musa J.D. (1999), Software Reliability Engineering: More Reliable Software, Faster Development and Testing, McGraw-Hill.

[13] Musa, J.D., Iannino, A. and Okumoto, K. (1987), Software Reliability: Measurement, Prediction and Application, McGraw-Hill.

[14] Ohba, M. (1984), "Software Reliability Analysis Models" IBM Journal. Research Development, Vol. 28, no. 4 , pp. $428-443$.

[15] Ohba, M. (1984a), "Inflection S-shaped Software Reliability Growth Models", Stochastic Models in Reliability Theory (Osaki, S. and Hatoyama, Y. Editors), pp. 144-162, Springer-Verlag, Merlin.

[16] Pham, H. (2000), Software Reliability, Springer-Verlag, New York.

[17] Quadri, S.M.K., Ahmad, N., and Farooq, S.U. (2011), "Software Reliability Growth Modeling with Generalized Exponential Testing-effort and Optimal Software Release Policy", Global Journal of Computer Science and Technology, Vol. 11 (2), pp. $26-41$. 
[18] Xie, M. (1991), Software Reliability Modeling, World Scientific Publication, Singapore.

[19] Yamada, S., Hishitani J. and Osaki, S. (1993), "Software reliability growth model with Weibull testing-effort: a model and application", IEEE Transactions on Reliability, Vol. R-42, pp. 100-105.

[20] Yamada, S., Ohba, M. and Osaki, S. (1984), "S-shaped Software Reliability Growth Models and their Applications", IEEE Transactions on Reliability, Vol. R33, pp. 289-292.
[21] Yamada, S. and Ohtera, H. (1990), "Software Reliability Growth Models for Testing Effort Control", European Journal of Operational Research, Vol. 46, 3, pp. 343-349.

[22] Yamada, S., Ohtera, H. and Norihisa, H. (1986), "Software Reliability Growth Model with Testingeffort", IEEE Transactions on Reliability, Vol. R-35, no. 1, pp.19-23.

[23] Yamada, S., and Osaki, S. (1985), "Software Reliability Growth Modeling: Models and Applications", IEEE Transaction on Software Engineering, Vol. SE-11, no. 12, pp. 1431-1437. 